\title{
Estimation of biophysical and functional properties of artery walls from pulse wave measured by photoplethysmography
}

\author{
M. Huotari, K. Määttä \& J. Kostamovaara \\ Department of Electrical and Information Engineering, \\ Electronics Laboratory, University of Oulu, Finland
}

\begin{abstract}
Arterial pulse wave analysis in time and frequency domain was carried out to find out biophysical and functional properties of artery walls measured with a photoplethysmographic (PPG) device. Because peripheral arterial disease (PAD) is a fatal problem all over the world, an easy diagnosis method would be needed. It could probably be diagnosed by PPG which is a non-invasive optical technique for detecting the arterial pulse waves. We present a study to characterize and quantify the arterial pulse wave components based on the use of logarithmic normal function (LNF). The measurements were carried out parallel from the index finger and toe tip with healthy subjects. In addition, a second derivative of the PPG signal (SNPPG) was also analyzed. The tests were applied to arterial pulse waves from 11 subjects between 5 and 69 years. The results show good correlation of pulse wave changes as a function of age.
\end{abstract}

Keywords: arterial stiffness, photoplethysmography, pulse wave analysis, percussion, tidal, dicrotic, pre-ejection wave component.

\section{Introduction}

Arterial stiffness has been estimated with many different analysis and procedures. One of them is the contour analysis of pulse waves. It has been proposed as a non-invasive means in assessing arterial stiffness in atherosclerosis. Accurate determination of the conventional parameters is usually precluded by changed waveforms in the aged and atherosclerotic objects. In this paper we introduce a new photoplethysmographic (PPG) way to estimate arterial elasticity or stiffness value. It has good reproducibility and it can also correlate 
with common atherosclerosis risk factors. According to our measurement subjects with higher age have very different values as PPG indexes.

Among the many methods used for pulse wave analysis, stiffness index (SI) and reflection index (RI) have been reported to be the two reliable indices for determining stiffness of large arteries. These indices do not take account of complete structure of the pulse wave [1]. They are based on the time interval between the blood pressure systolic and diastolic peaks and ratio of their heights. However, these parameters may be distorted by the other wave components which also belong to the complicated pulse wave structure. SI and RI can be difficult to determine in the ill-defined waveforms of elder people, for example.

The light energy absorption of blood within the visible and infrared regions is partly caused by the oxidized and reduced hemoglobin. The PPG measures the blood flux in human vessels with means of red and infrared light absorption. The absorption of light varies with the oxygen concentration and amount of blood in vessels but also with the vessel wall movement. Our PPG device is based on phase sensitive detection electronics which has proved to be a good solution for the measurement of small changes in the absorption and transmission of light signals simultaneously at two different wavelengths, 660 and $940 \mathrm{~nm}$. The PPG waveforms or simply pulse waves can be rapidly acquired with a PIN photodiode which measures the transmission of red and infra-red LED light simultaneously through the forefinger and the second toe. The waveforms have different characteristics for the young and elderly persons. Our suggestion is to decompose the measured waveform into four or five primary components to improve the accuracy of the analysis, and then to use a parameter calculated from the mutual time. Positions of the individual components characterize the arterial stiffness. In the wave analysis, the first wave is called a percussion wave, the second is a tidal, the third is a dicrotic, and the fourth and fifth are pre-ejection waves.

The PPG measurements may provide a cheap, simple and accurate method of diagnosing arterial and vascular diseases. Moreover, further development of the theoretical model that correlates the waveform of the detected finger and toe tip waves caused by heartbeat oscillations and the hemodynamic parameters could improve the accuracy of the method and potentially lead to a better quantification of the measured parameters used for arterial stiffness.

\section{Materials and methodology}

When part of the forefinger's and the second toe's nail is illuminated, it is possible to obtain a PPG waveform. PPG technique has not been fully validated except that it measures many hemodynamic parameters, which are not yet exactly known. To obtain a minimum noise PPG, we chose the phase sensitive detection principle based on the transmission probe. In practice, all human subjects were recorded in the supine position with a total measurement time of $300 \mathrm{~s}$ PPG probes placed on the left index finger and on the second toe. The infrared LED light has a peak wavelength of $940 \mathrm{~nm}$. To eliminate motion artifact, the subjects were encouraged to keep their fingers and toes relaxed. The 
data were collected over the bandwidth of $200 \mathrm{~Hz}$ and the integration, derivation, and spectrum calculation done with a digital signal analyzer. The raw data (ECG, phonocardiography (PCG), PPG1, PPG2) and calculated data files were transferred to a PC for later analysis. Baseline restoration was performed in sequential 10 to 12 pulse waves selected for decomposition analysis.

One potential technique for waveform decomposition is LevenbergMarquardt optimization algorithm (LMO) with non-linear fitting. Preliminary trial of the LMO technique was performed on a PPG data recorded from the elderly and young subjects. With the young subjects, e.g., radial wave is easy for analysis purposes, whereas with the elderly, it is very difficult to analyze completely. When studying wave reflections, it is possible to decompose the measured PPG waveform into the percussion wave and the four reflected components, e.g., the tidal, the dicrotic, and the peripheral reflection waves for necessary index calculation. For the accurate determination of the time of arrival of the reflected waves we realized a trial and error procedure. This analysis can be done in the time domain. This technique has its pros and cons, but the final fitting result based on the residual error and prediction band gave good results.

\section{Results and discussion}

Figure 1 presents the actual measurement results from a male subject with the age of 25 years. It should be noted that the PPGs are downward because of the absorption. Figure 2 shows the PPG1 waveform separately and Figure 3 its second derivative.

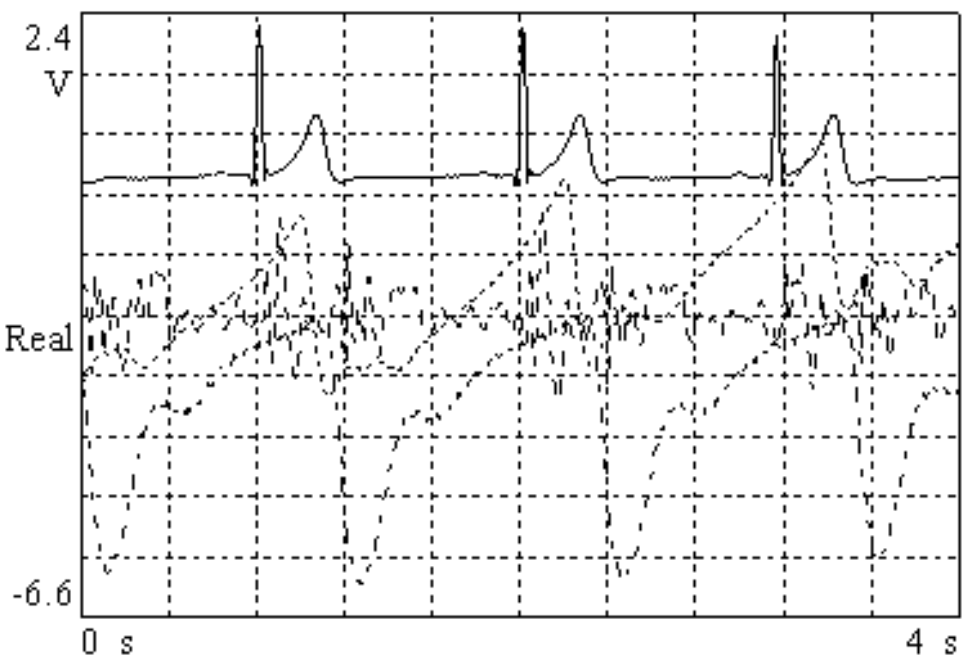

Figure 1: Part of the actual measurement results (4 s): ECG (line), PCG (dash), PPG1 (finger, dots), and PPG2 (toe, dash dots) with a male subject, aged 25 years. 


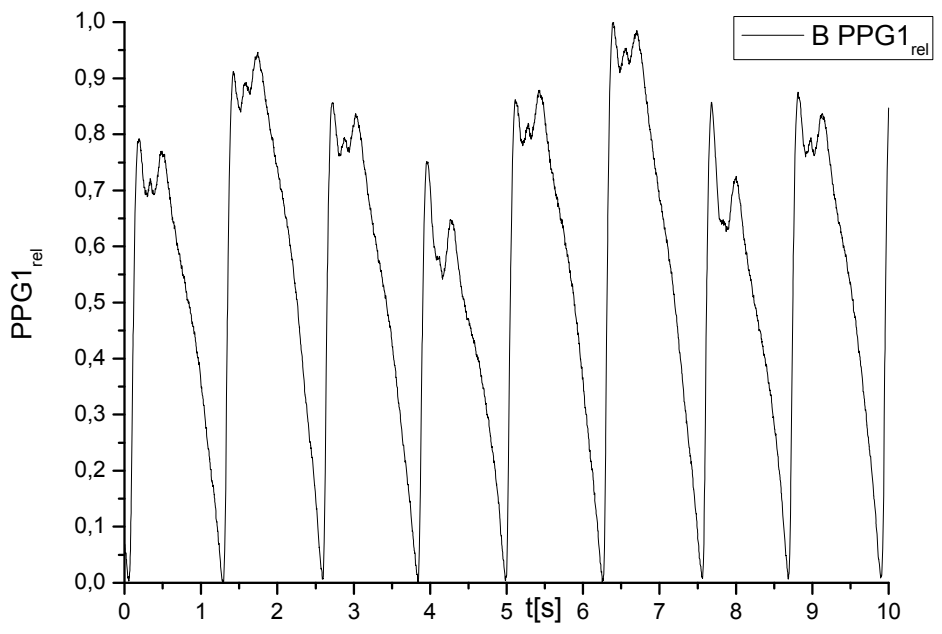

Figure 2: $\quad$ PPG1 during 10s (8 pulse waves of finger) after baseline correction in Figure 1 it is original PPG1 (finger, dots).

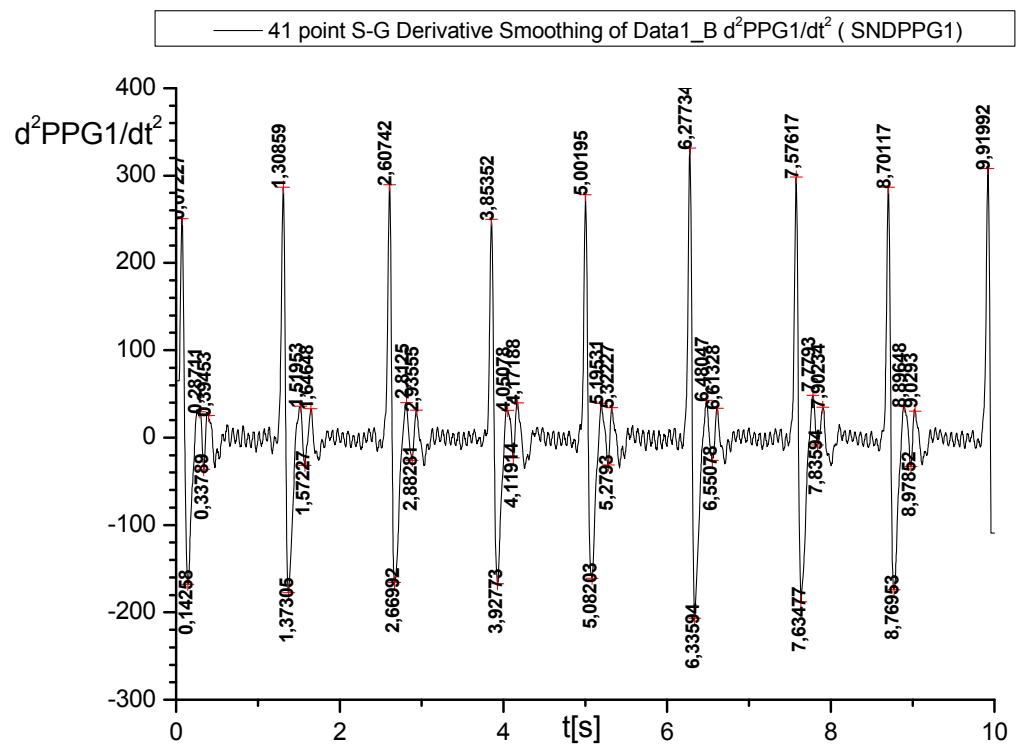

Figure 3: $\quad$ The second derivative of the measured PPG1 during 10s.

From the second derivative of the photoplethysmogram the amplitudes of the all five peaks (A, B, C, D, and E) are easily determined. 

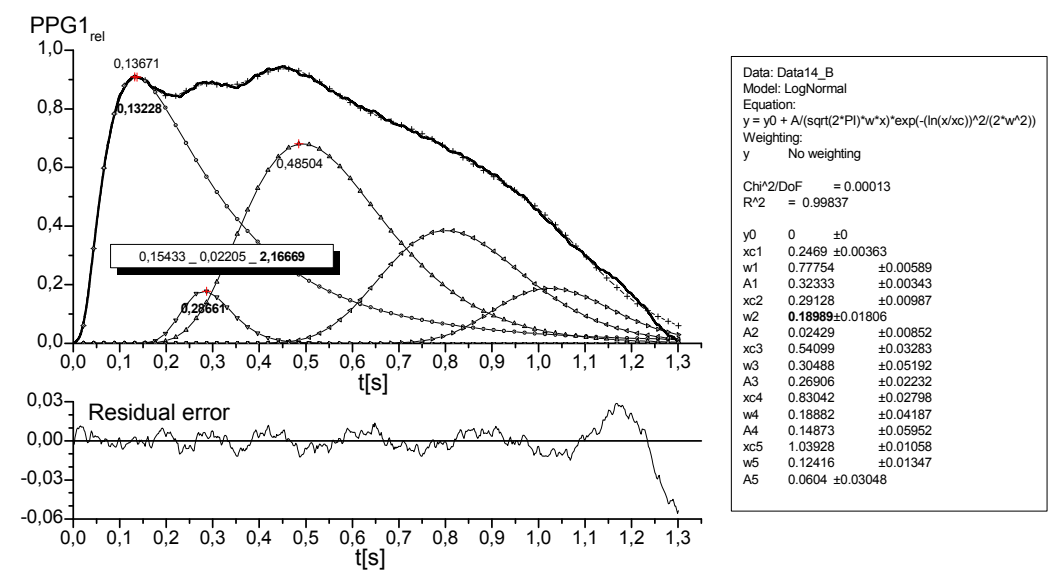

Figure 4: The decomposed PPG1 of Figure 3. It is seen that the tidal component peak time position divided by the percussion component peak time position is 2.1666 .

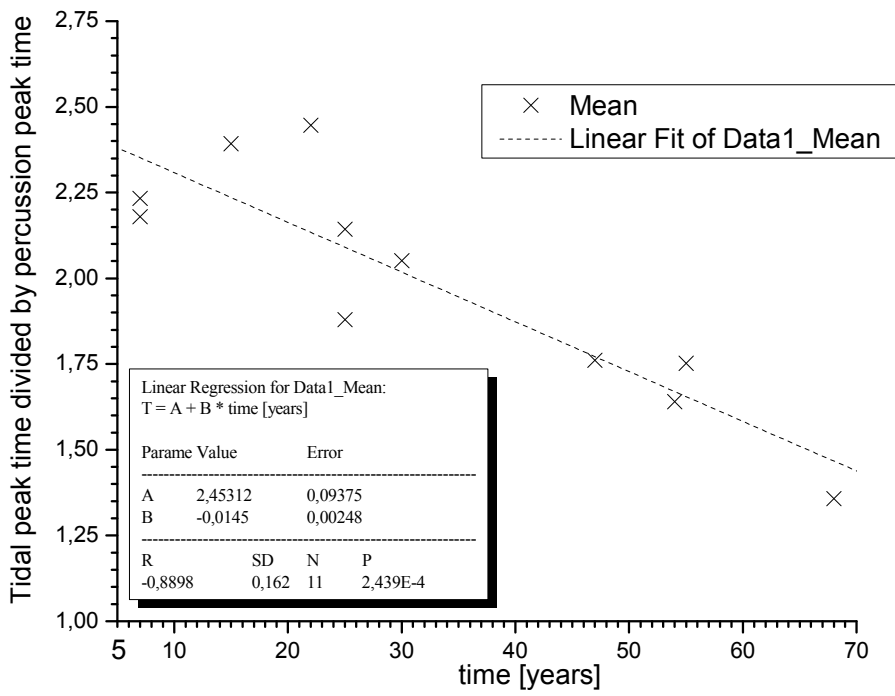

Figure 5: The dependence of the suggested parameter as a function of the age of the subjects under study. It is shown the correlation coefficient is over 0.8 for this small group of healthy persons whose ages are between 7 and 68 years. 
Figure 4 depicts the decomposed photoplethysmogram pulse. In this specific pulse the time position of the tidal wave peak with respect to time zero is about two times as large than with the percussion peak time. We suggest that this parameter could probably be related to arterial elasticity and could correlate with the severity of atherosclerosis. Elastic characteristic becomes from the capability of arterial walls being distended or stretched under blood pressure pulse. This makes it an important biophysical property. The dependence of this parameter on age is depicted in Figure 5.

Figure 6 depicts the integrals of ECG, PPG1 and PPG2, respectively. They all have the similar trends, however the ECG integral contains higher frequencies than the PPG integrals.

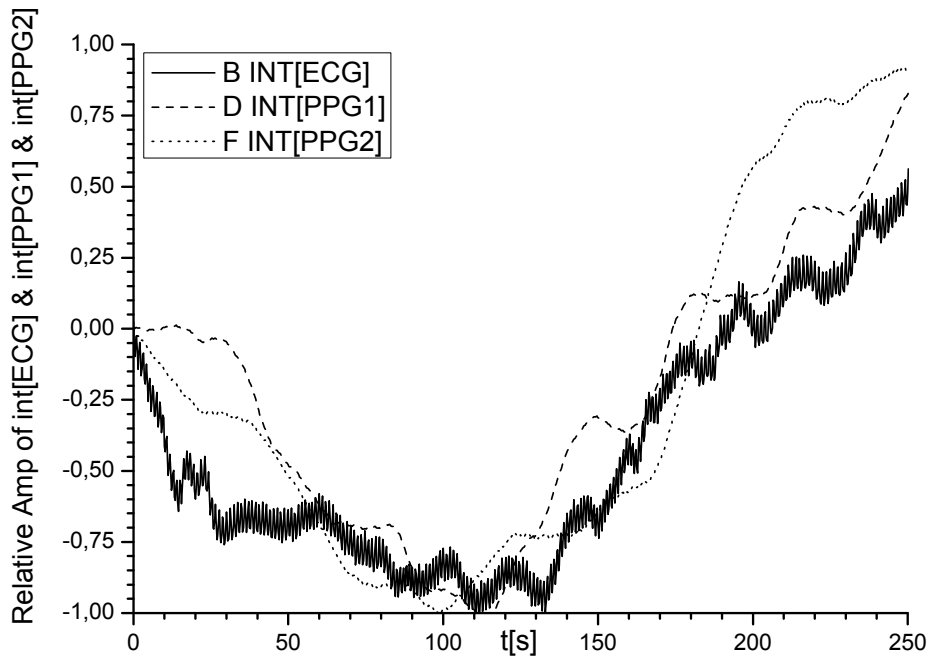

Figure 6: The integrated ECG, PPG1, and PPG2 signals.

The integrals of PPGs are the true DC signal part in PPG measurements. They are generated by autonomic neural system [2]. Each pulse wave is the AC signal part of PPG responsible of e.g. arterial elasticity. An advantage of the PPG measurement and its pulse wave analysis is that the PPG pulse waves can be obtained easily without electrodes, thus making this device useful for epidemiological applications. In this study we are especially interested in the genesis of tidal pulse wave component which is generated after the opening of the aortic valve. The pulse propagates through the aorta storing elastic energy in the aortic wall which is causing the tidal wave phenomena. After the closing the aortic valve the elastic energy of the aorta is recoiled as the tidal component recorded clearly as a part of the PPG waveform in the finger tip. However, the toe tip PPG (=PPG2) contains only traces of the tidal wave component in the second derivative PPG. 


\section{Conclusion}

PPG is a noninvasive method to study peripheral arterial responses, such as pulse waveforms, autonomic fluctuations, and peristaltic of intestine. Simultaneous recording of the ECG and PCG (phonocardiogram) were done in this study for the better interpretation of the PPG pulse wave results. The application of a PPG device with five logarithmic normal functions for the finger PPG waveform analysis gave good accuracy in this small subjects group. In a selected diseased subject group, it could be possible to estimate arterial conditions, e.g. arterial stiffness compared with healthy cohorts. Because the pulse wave characteristics in peripheral pulse depend on the propagation conditions in the arteries, we applied pulse wave decomposition with the hope of a low-cost and noninvasive monitoring system to recognize hidden arterial diseases. PPG could have potential in the so called preventive monitoring of elderly people's health and home care conditions.

\section{Acknowledgement}

The present study has been carried out with the support to $\mathrm{MH}$ from the Finnish Cultural Foundation.

\section{References}

[1] S. C. Millasseau et al. (2006) Contour analysis of the photoplethysmographic pulse measured at the finger Journal of Hypertension, Vol 24 No 8.

[2] S. M. Yacin et a1. (2010) On Non-Invasive Measurement of Gastric Motility from Finger Photoplethysmographic Signal. Annals of Biomedical Engineering, Vol. 38, No. 12, pp. 3744-3755. 